\title{
RESTAURACIÓN CON INCRUSTACIÓN SEMIDIRECTA DE RESINA EN UNA MISMA SESIÓN: CONCEPTO CHAIRSIDE
}

Restoration with semi-direct resin inlay in the same session: Chairside Concept

Fecha de Recepción: 4 de noviembre 2020
Restauração com semi-direct resin inlay na mesma sessão: Chairside Concept

Aceptado para su publicación: 30 de noviembre 2020

\section{Autores: \\ Daniel P Rosa1,a Demetrio O Kulgawczuk ${ }^{1, b}$ Joaquín H Jahke ${ }^{1, c}$ Martin R Pratto ${ }^{1, c}$ Jorge E Aredes ${ }^{1,2, d}$}

1. Carrera de Especialización en Prótesis Dentobucomaxilar. Facultad de Odontología. Universidad Nacional del Nordeste. República Argentina.

2. Cátedra de Clínica I de Prótesis. Facultad de Odontología. Universidad de Buenos Aires. República Argentina.

a. Doctor de la Universidad Nacional del Nordeste en Odontología (UNNE)

b. Especialista en Prótesis Fija, Removible e Implantología (Universidad Nacional de Córdoba) c. Odontólogo (UNNE)

d. Doctor de la Universidad de Buenos Aires, Área Odontología Rehabilitadora

Correspondencia:

Rosa, Daniel Pablo Av. Libertad 5450, W3400 Corrientes +540379445-7992

Correo electrónico: danielrosa10@hotmail.com danielpablorosa@gmail.com

Conflicto de intereses: los autores declaran no tener conflictos de interés.

Fuente de financiamiento: autofinanciado.

\section{Resumen}

Las restauraciones indirectas de resina compuesta son una alternativa actual para mejorar el desempeño clínico en grandes reconstrucciones. Pueden ser realizadas de forma rápida y simple en el consultorio por el odontólogo, en una misma sesión junto a la preparación dentaría, por eso se denominan semidirectas y entran en la categoría de tratamiento chairside. Son considerados tratamientos más rápidos, menos costosos, y sin la intervención del técnico dental. El objetivo de este caso clínico fue presentar la rehabilitación de la pieza 3.6 con caries extensa, en un paciente masculino de 22 años de edad, mediante la construcción de una incrustación en resina compuesta de foto activación. Con la incrustación semidirecta de resina compuesta en una misma sesión, podemos lograr excelentes resultados tanto estético como anátomo-funcionales, optimizando recursos económicos y de tiempo, permitiendo incluso accesibilidad al tratamiento en zonas distantes a los laboratorios dentales entrenados.

Palabras clave: restauración dental permanente, resinas compuestas, diente molar (fuente: DeCS BIREME).

\footnotetext{
Abstract

Indirect resin composite restorations are a current alternative to improve clinical performance in large reconstructions. They can be performed quickly and simply in the office by the dentist, in a single session along with the dental preparation, which is why they are called semi-direct and fall under the category of chairside treatment. They are considered faster, less expensive treatments, and without the intervention of the dental technician. The objective of this clinical case was to present the rehabilitation of piece 3.6 with extensive caries,
} 


\section{REFO}

VOL. XIII | N² 2| AÑO 2020

ISSN 1668-7280

ISSN-E 2683-7986 in a 22-year-old male patient, by means of the construction of a composite photo-activation resin inlay. With the semi-direct inlay of composite resin in the same session, we can achieve excellent results both aesthetical and anatomical - functional, optimizing economic resources and time, even allowing accessibility to treatment in distant areas from trained dental laboratories.

Key words: permanent dental restoration, composite resins, molar (source: MeSH NLM).

\section{Resumo}

As restaurações indiretas de resina composta são uma alternativa atual para melhorar o desempenho clínico em grandes reconstruções. Podem ser realizados de forma rápida e simples no consultório odontológico pelo dentista, na mesma sessão junto com o preparo dentário, por isso são chamados de semi-diretos e se enquadram na categoria de tratamento chairside. São considerados tratamentos mais rápidos, mais baratos $e$ sem a intervenção do técnico dentário. O objetivo deste caso clínico foi apresentar a reabilitação do dente 3.6 com cárie extensa, em um paciente do sexo masculino, 22 anos, por meio da confecção de um inlay de resina composta de fotoativação. Com o inlay semidireto de resina composta na mesma sessão, podemos alcançar excelentes resultados estéticos e anátomo-funcionais, otimizando recursos econômicos e de tempo, permitindo inclusive acessibilidade para tratamento em áreas distantes de laboratórios odontológicos treinados.

Palavras-chave: restauração dentária permanente, resinas compostas, dente molar (fonte: DeCS BIREME).

\section{Introducción}

La restauración de piezas dentarias, mediante el uso de resinas compuestas de modo directo está indicada en aquellos casos clínicos con moderada pérdida de sustancia. Representan sin duda la opción más funcional y conservadora para la restitución de la estructura dentaria perdida. Sin embargo, cuando se realizan las reconstrucciones directas de resina compuesta en dientes con extensa pérdida de estructura, existen dificultades técnicas que pueden ejercer una negativa influencia en el resultado final, afectando la longevidad de las restauraciones.
Es difícil trazar una línea exacta entre la posibilidad de realizar una restauración con técnica directa o indirecta. Por otra parte, en términos de longevidad, las dos alternativas ofrecen resultados similares $^{1}$.

Las restauraciones indirectas de resina compuesta, surgen con la intención de mejorar el desempeño clínico de las resinas compuestas directas. Pueden ser confeccionadas por los técnicos de laboratorio, como también pueden ser realizadas de forma rápida y simple en el consultorio por el propio odontólogo, en este caso se denominan semidirectas. A su vez, cuando se realizan en una misma sesión junto con la preparación dentaría, entran en la categoría de tratamiento chairside, que implica que fue realizado junto al sillón.

De esa forma, a través de la restauración dentaria indirecta, fuera del ambiente bucal, es posible reducir los efectos perjudiciales de la contracción de polimerización, aumentando el grado de conversión de los monómeros resinosos en polímeros, además de mejorar las condiciones clínicas de trabajo. Así, la reconstrucción del punto de contacto proximal, la adaptación de los márgenes de la restauración, la caracterización y la escultura anatómica, además de las etapas finales de acabado y pulido, se pueden controlar de forma más eficaz. Al ser confeccionadas por el odontólogo, sobre un modelo y fuera de la cavidad bucal, se reducen las dificultades técnicas que presenta el trabajo intraoral, teniendo mejor visión, acceso y comodidad de trabajo.

El uso de modelos no rígidos en la ejecución de restauraciones inlay y onlay, facilitan la construcción de piezas de forma extraoral, sin la necesidad de una segunda sesión, desde el momento en que la polimerización de estos modelos es lo suficientemente rápida. La previsibilidad del resultado es garantizada por una excelente combinación de moldes realizados con alginatos y modelos elaborados con materiales a base de silicona, o bien de impresiones de silicona y modelos de poliéster ${ }^{2-4}$.

El concepto de tratamiento protético en una misma visita y de resolución integra dentro del consultorio odontológico, de allí el término "al lado del sillón" o chairside, es una idea desarrollada hace 
muchos años ya, en la búsqueda de obtener tratamientos más rápidos y con menor costo, sin la intervención del técnico dental. Esto se vuelve especialmente importante cuando por razones locales se dificulta acceder a los servicios de un técnico de laboratorio, ya sea por distancia, tiempos o costos. La técnica utilizada en este trabajo requiere del manejo por parte del odontólogo tratante de los materiales y técnicas para poder confeccionar la pieza protética en su consultorio, en contraposición con aquellos trabajos que se envían al laboratorio dental para su elaboración, a los que llamaremos trabajos Labside. Los trabajos chairside, ya sea elaborados por técnicas manuales tradicionales pero simplificadas, como se exponen en esta publicación, o bien asistidas por técnicas digitales (impresiones digitales y manufactura CAD-CAM), tienen por objetivo permitir al odontólogo la resolución rápida y simplificada de situaciones clínicas de la práctica diaria, con un alto nivel de calidad y control total del proceso ${ }^{2,5}$.

\section{Caso Clínico}

El presente trabajo fue realizado dentro del marco de la carrera de Especialización en Prótesis Dentobucomaxial de la FOUNNE. Se presenta un paciente masculino de 22 años de edad, que concurrió a la consulta para un control general, luego de realizar la Historia Clínica General y Odontológica, es derivado al servicio con una lesión de caries extensa (Mesio-Ocluso-Distal) en la pieza dentaria número 36 y ausencia de la pieza dentaria número 37.

Después de un examen radiográfico de la pieza y por las características clínicas del caso, que involucraba una extensión importante de la pieza dentaria afectando la relación de contacto con la pieza dentaria número 35, se optó por la técnica de incrustación semidirecta de resina.

Se procedió a realizar una anestesia infiltrativa troncular al nervio dentario inferior. Se realizó aislamiento absoluto con dique de goma de las piezas número 34, 35, 36 y la posterior eliminación del tejido cariado, utilizando intermitentemente detector de caries, caries indicator (Ultradent Products, Inc.). Luego se preparó la pieza de modo expulsivo para recibir la restauración y verificando que los grosores de las cúspides remanentes sean iguales o ma- yores a $2 \mathrm{~mm}^{5-7}$. Concluida la eliminación de todo el tejido afectado y la realización de la preparación, se procedió a realizar una técnica de sellado dentinario inmediato para rellenar zonas retentivas o de socavado $^{2,8}$, (Figura 1). Con una piedra troncocónica de punta plana y ángulos redondeados (846R - Jota AG) se dio la forma de conveniencia a la preparación.

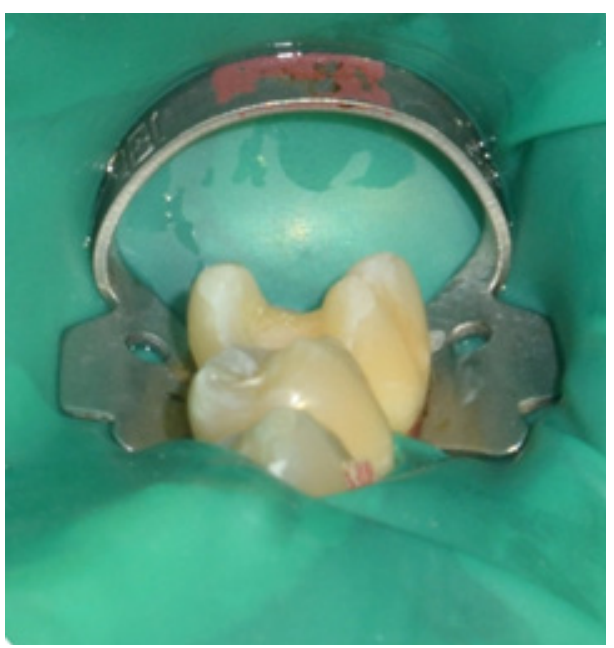

Figura 1. Eliminación de caries, material de obturación antiguo y acondicionamiento del tejido dentario.

Luego de retirar el aislamiento, se procedió a tomar una impresión con alginato (Jeltrate ${ }^{\circledR}$ Plus, Dentsplay) corroborando la calidad de la impresión se procedió a vaciar la misma con silicona por adición de consistencia liviana y regular (President ${ }^{\circledR}$, Coltene). Polimerizada la silicona, en pocos minutos, se obtuvo el modelo de trabajo. También, se realizó luego de retirar el modelo de silicona un vaciado en yeso densita tipo IV, para poder corroborar el calce y ajuste de la incrustación. Mientras sobre él primer modelo se construía la restauración final no fue necesario colocar ningún material provisorio, ya que todo el procedimiento lo realizamos en la misma sesión.

Para construir la incrustación se utilizó una resina compuesta de foto activación desarrollada específicamente para este fin (SR Nexco, Ivoclar Vivadent). Sobre un liner previo, se colocó una fina capa de resina denominada oclusal dentin orange y luego sus diferentes capas dentina A2 y A3, esmalte color A1 y traslúcido como última capa, de la escala Vita Clasic y luego se cubrió con gel para su polimerización final. Se polimerizó en un horno de fabricación 


\section{REFO}

VOL. XIII | N² 2| AÑO 2020

ISSN 1668-7280

ISSN-E 2683-7986 nacional, marca Cube, que provee una intensidad de luz superior a 7.000 lumens. El pulido se realizó con gomas para pulir composite (Jota AG) de uso habitual en operatoria dental y por ultimo con cepillo y disco de algodón con pasta de alto brillo. Paso siguiente se confirmó el calce y ajuste de la incrustación en el modelo de yeso, (Figura 2).

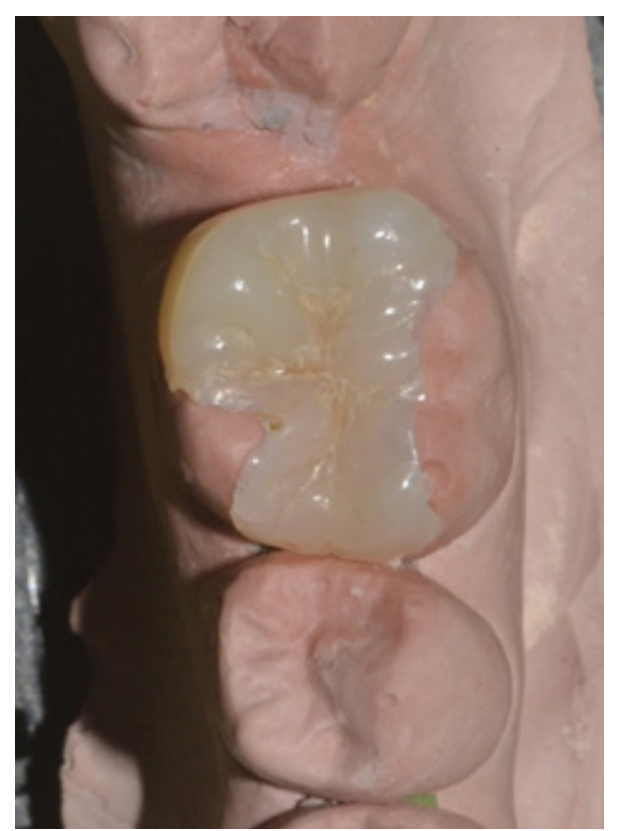

Figura 2. Control de calce y ajuste de la incrustación en el modelo de yeso densita.

Para su cementación se procedió a realizar aislamiento absoluto del campo operatorio, se probó el asentamiento y adaptación de la restauración en boca y se preparó la pieza para su cementación. El interior de la restauración se asperiza con piedra fina y luego se silaniza (Monobond $®$ Plus - Ivoclar Vivadent) para facilitar la unión química. La superficie dentaria, a la que se le realizo técnica de sellado dentinario inmediato post preparación, se prepara mediante aplicación de ácido fosfórico al $37 \%$ y luego se utilizó en este caso un cemento dual (RelyX ${ }^{\mathrm{TM}}$ $\mathrm{U} 200,3 \mathrm{M}^{\mathrm{TM}}$ ). Se polimerizó 5 segundos en cada cara, se extrajo con explorador los excedentes y luego se completó la polimerización, 40 segundos en cada cara. Retirado el aislamiento absoluto se procedió al control final de la oclusión y pulido de los excesos en la interface restauración-diente, (Figura 3). Se controló al mes, corroborando la ausencia de sensibilidad postoperatoria.

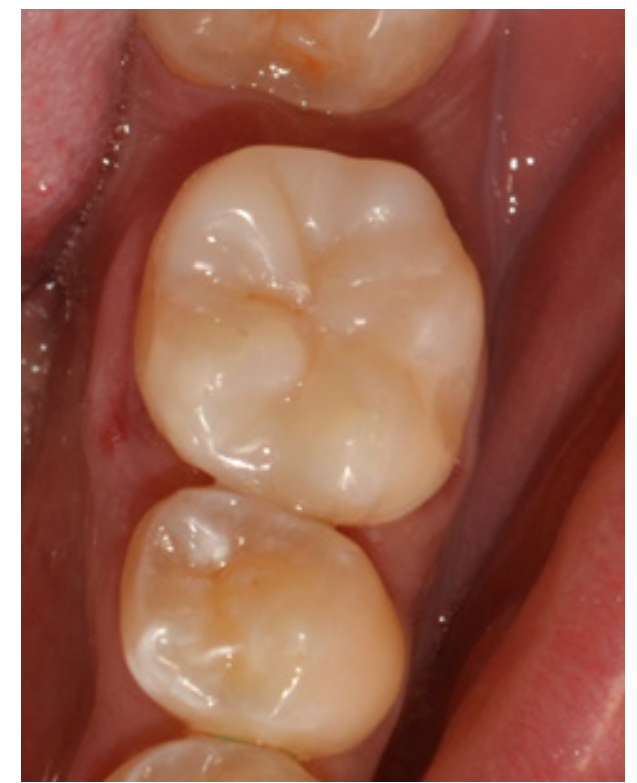

Figura 3. Restauración finalizada.

\section{Discusión}

La técnica presentada, en algunos casos puede presentar un límite difuso, en lo referido a su aplicación frente a la chance de inclinarnos por una técnica directa. Dadas las condiciones y habilidad de cada profesional, ambas pueden conducir a un excelente resultado. Aun así, son numerosos los benéficos que nos brinda el manejo de las restauraciones indirectas, sumado a la alternativa de la realización total del tratamiento en una misma sesión. La mejora de las características físicas del material, la simplicidad de manejo de la morfología y modelado extraoral, la rapidez de solución para el paciente, la integración de los materiales mediante el uso de técnicas adhesivas y la combinación con la técnica de sellado dentinario inmediato, son todas ventajas que redundan en una mejora integral en la práctica profesional ${ }^{1,2}$.

Su realización requiere que se identifiquen los factores sensibles e importantes que deben ser tenidos en cuenta: una preparación dentaria correcta, conservadora y expulsiva, el proceso de elaboración de la incrustación respetando las indicaciones del fabricante de los materiales y la fijación adhesiva de la misma a la pieza dentaria, con un manejo correcto de los protocolos adhesivos?.

Las ventajas de este procedimiento las podemos resumir en: 1) control de la contracción de polimerización, 2) mejora de propiedades físicas, 3) co- 
rrección del modelado y contorneado de la pieza, 4) facilita la accesibilidad y disminuye tiempo clínico, 5) beneficio económico, 6) independencia del laboratorio dental, 7) no requiere uso de provisorios, 8) perfecta integración con el medio cementante adhesivo y los materiales de relleno ${ }^{8.9}$.

En conclusión, con la técnica descripta de incrustación semidirecta de resina en una misma sesión, podemos lograr excelentes resultados tanto estético como anatomofuncionales, optimizando recursos. También nos facilita las maniobras operatorias, permite mejorar las propiedades del material y el sellado de la restauración, brindando una alternativa de rehabilitación en una sesión, con la consiguiente ventaja de poder solucionar dentro del marco del consultorio dental situaciones puntuales como la planteada en este trabajo.

\section{Referencias Bibliográficas}

1. Spreafico RC. Composite resin rehabilitation of eroded dentition in a bulimic patient: a case report. Eur J Esthet Dent. 2010 Spring;5(1):28-48.

2. Hirata R. TIPS: dicas em odontologia estética. Brasil: Panamericana Publishing Co. Inc.;2011.

3. Gerrow JD, Price RB. Comparison of the surface detail reproduction of flexible die material system. J Prosthet Dent.1998;80(4):485-9. DOI: 10.1016/s00223913(98)70016-2

4. Price RB, Gerrow JD. Margin adaptation of indirect composite inlays fabricated on flexible dies. J Prosthet Dent. 2000;83(3):306-13. DOI: 10.1016/s00223913(00)70133-8

5. Krifka S, Anthofer T, Fritzsch M, Hiller KA, Schmalz G, Federlin M. Ceramic inlays and par-tial ceramic crowns: influence of remaining cusp wall thickness on the marginal integrity and enamel crack formation in vitro. Oper Dent. 2009;34(1):32-42. DOI: 10.2341/08-34

6. Fennis WM, Kuijs RH, Barink M, Kreulen CM, Verdonschot N, Creugers NH. Can internal stresses explain the fracture resistance of cusp-replacing composite restorations? Eur J Oral Sci. 2005;113(5):443-448. DOI: 10.1111/j.1600-0722.2005.00233.x

7. Fennis WM, Kuijs RH, Kreulen CM, Verdonschot $\mathrm{N}$, Creugers NH. Fatigue resistance of teeth restored with cuspal-coverage composite restorations. Int J Prosthodont. 2004;17(3):313-317.

8. Sinjari B, D’Addazio G, Xhajanka E, Caputi S, Varvara G, Traini T. Penetra-tion of Different Impression Materials into Exposed Dentinal Tubules during the Impression Procedure. Materials (Basel, Switzerland). 2020;13(6):1321. DOI: 10.3390/ma13061321

9. Rocca GT, Rizcalla N, Krejci I, Dietschi D. Evidence-based concepts and procedures for bonded inlays and onlays. Part II. Guidelines for cavity preparation and restoration fabrication. Int J Esthet Dent. 2015;10(3):392-413. 